\title{
Fecal Bile Acids and Fecal Short Chain Fatty Acids in Patients with Irritable Bowel Syndrome and Control Volunteers
}

\author{
Chirag Patel ${ }^{1}$, Carolyn Lockett ${ }^{2}$, Huiping $\mathrm{Xu}^{3}$, Andrea Shin ${ }^{2}$ \\ ${ }^{1}$ Indiana University School of Medicine, ${ }^{2}$ Division of Gastroenterology and Hepatology, \\ ${ }^{3}$ Department of Biostatistics, Indiana University School of Medicine
}

Background and Aims: Fecal bile acids (BAs), short chain fatty acids (SCFAs), and gut microbiome may be implicated in irritable bowel syndrome (IBS) pathophysiology. Our aim was to compare fecal organic acids between IBS with constipation (IBS-C), IBS with diarrhea (IBSD), and controls.

Methods: Stool samples were collected from 17 controls, 5 IBS-C, and 5 IBS-D volunteers during a 4-day high fat diet. Aliquots were stored for future analysis of the fecal microbiota. Fecal SCFA and BA analyses were conducted at the Metabolite Profiling Facility at Purdue University and Laboratory Medicine and Pathology at Mayo Clinic. We compared SCFA and BA levels among groups using the Wilcoxon rank sum test. Gamma and linear regression were used to compare SCFAs and BAs adjusting for age and body mass index (BMI).

Results: Fecal acetate levels (mean+SD, $\mu \mathrm{g} / \mathrm{mg}$ ) were higher in IBS-C $(11.3 \pm 7$ ) than in controls (6.1 \pm 3.3$)$ or IBS-D $(7.7 \pm 2)$, although not statistically significant $(p=0.19)$. Total fecal BAs (median [IQR], \%) were higher in IBS-D (675 [484-778]) than in controls (342 [130-640]) or IBSC (321.5 (34.5-718); however, differences were not significant. No significant differences were observed in BAs or SCFAs between groups in multivariate analyses.

Conclusion: We are unable to show significant differences in organic acid levels in IBS and controls. Lack of association may be due to small sample size. Future investigation of larger patient numbers with incorporation of transit and microbiome analyses may shed further light on the role of organic acids in IBS to identify new biomarkers and treatment targets. 